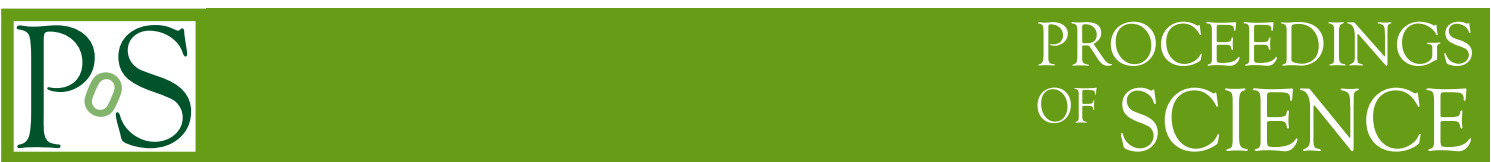

\title{
Galaxy-scale maser astrometry with VERA
}

\author{
Mareki Honma* \\ Mizusawa VLBI Observatory, NAOJ, Mitaka, Tokyo 181-8588 \\ Graduate University for Advanced Study, Mitaka, Tokyo 181-8588 \\ E-mail: mareki.honma@nao.ac.jp
}

In this paper we summarize most updated results from VERA (VLBI Exploration of Radio Astrometry). VERA has been conducting regular monitoring of Galactic radio sources since 2004, and we have already measured parallaxes for $\sim 30$ maser sources. Based on combination of highaccuracy astrometry by VERA, VLBA and EVN, the fundamental parameters of the Milky Way Galaxy are determined. While the Galaxy center distance is consistent with recent other studies, the Galactic rotation velocity based on high-accuracy astrometry suggested that the Galactic rotation and hence the mass of the Galaxy requires an upward modification.

11 th European VLBI Network Symposium \& Users Meeting,

October 9-12, 2012

Bordeaux, France

${ }^{*}$ Speaker. 


\section{VERA and its current status}

VERA (VLBI Exploration of Radio Astrometry) is a VLBI array in Japan dedicated to phasereferencing astrometry. VERA conducts astrometric observations of Galactic maser sources such as star-forming regions and AGB (Asymptotic Giant Branch) stars. Based on accurate distances and proper motions of maser sources, VERA will reveal the three-dimensional structure and dynamics of the Milky Way Galaxy. The VERA array consists of four 20-m diameter telescopes with the maximum baseline length of $2300 \mathrm{~km}$. The main observing bands are: $6.7 \mathrm{GHz}$ for methanol masers, $22 \mathrm{GHz}$ for $\mathrm{H}_{2} \mathrm{O}$ masers, $43 \mathrm{GHz}$ for $\mathrm{SiO}$ masers. The most unique feature of VERA is that it has dual-beam system to observe a target maser source and reference extra-galactic source at the same time to effectively cancel out tropospheric fluctuation. Construction of the VERA array was completed in 2002, and the first astrometric results were published in 2007.

First astrometric results are obtained for star forming regions S269 [9] and Orion KL [5]. Since then, astrometry of Galactic maser sources has been conducted on regular basis, and astrometric results for more than 30 sources have been already published. Recent results are summarized in the two VERA special issues in PASJ in 2008 \& 2011 and references therein (e.g., [10, 6]). Most recently, in 2012, we have published several papers on astrometric results including IRAS 05168+3634 [21], RX Boo [13], IRAS 22480+6002 [12], and so on. Recent results related to VERA and JVN (Japanese VLBI Network, which includes VERA as a part) are also reported in this proceeding volume of the 11th EVN symposium such as [8], [24], [15], [22], [17], [19], and so on. The current status of VERA's astrometry is summarized in figure 1, showing the distributions of the maser sources for which astrometric measurements were obtained. As seen figure 1, for sources within $5 \mathrm{kpc}$ reliable parallaxes are readily available. For the distant sources beyond $5 \mathrm{kpc}$, it is still not easy to obtain reliable parallax, but accurate proper motions can be measured even for them.

In addition to maser astrometry, VERA is also used for mapping observations of AGN (Active Galactic Nuclei). One of the most notable example is the GENJI (Gamma-ray Emitting Notable AGN Monitoring by Japanese VLBI) project. The GENJI project utilizes the data of fringe finder sources (which are radio-bright gamma-ray sources such as BL Lac, 3C273, 3C279, 3C454.3, NRAO 530 etc.) during regular VERA operation, as in any observation fringe finder sources are observed every few hours to track clock parameter variation. Since the start of this project (late 2010), several sources have been monitored for a few year term, and comparison with gamma-ray data from Fermi satellite already produced interesting results related gamma-ray flaring activity (e.g., $[18,16])$.

\section{Galactic parameter determinations}

In addition to VERA, VLBA BeSSeL project [1] and also EVN have been producing highlyaccurate astrometric results. Using these astrometric data, we can determine the fundamental structure of the Milky Way Galaxy. Figure 2 shows the distribution of 52 star-forming regions for which accurate distances and proper motions are available (see [11]). As seen in figure 2, except in the southern Galaxy between $l=240^{\circ}$ and $350^{\circ}$, the sources are well-distributed over a wide range of Galactic longitude (from the Galactic center to the anti-center). The distribution of the 52 sources 


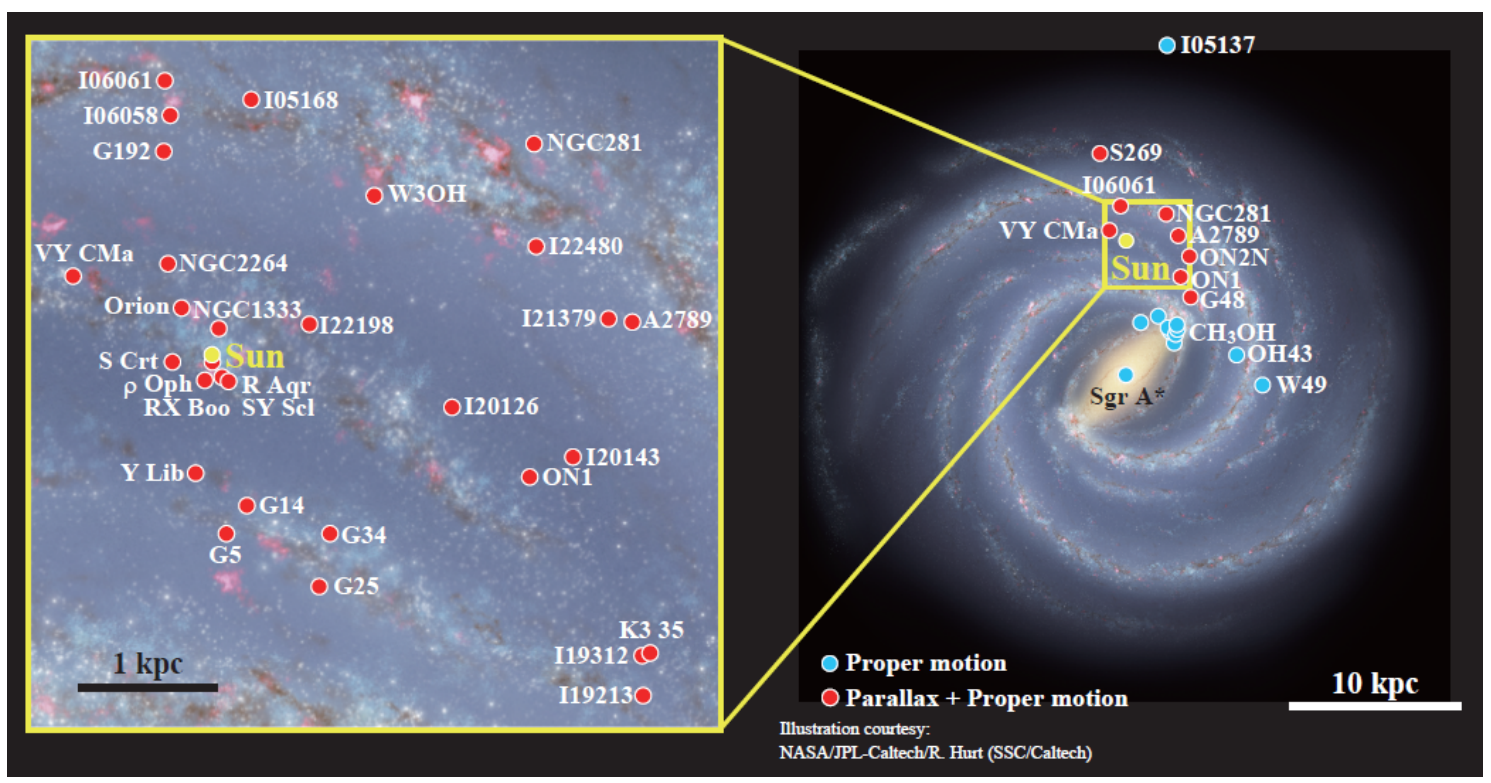

Figure 1: A schematic face-on view of the Milky Way Galaxy with locations of radio sources for which astrometric observations have been conducted with VERA. Red spots are those for which parallaxes and proper motions have been measured, and blue spots are those only with proper motion measurements.

in a face-on view is shown in figure 3 . We also show the observed proper motion vectors for these star-forming regions, from which one can easily see the Galactic rotation. For these sources, we have the full 6-dimensional phase-space information (three-dimension positions and velocities), and by constructing a Galaxy model to describe their motions, we can determine the fundamental parameters of the Milky Way Galaxy. Here we introduce a simple Galaxy model, in which we assume circular rotation with a power-law Galactic rotation curve. To describe the observed proper motions of star-forming regions, we use six parameters, namely, the Galactic center distance $R_{0}$, Galactic angular velocity $\Omega_{0}$, the rotation curve power-law index $\alpha$, and the mean peculiar motion of star-forming regions $\left(U_{\text {src }}, V_{\text {src }}, W_{\text {src }}\right)$. In order to survey such a multi-dimensional space effectively, we have utilized the MCMC (Markov Chain Monte Carlo) method to find the best Galactic parameters.

According to the MCMC, the best parameters are obtained as follows: $R_{0}=8.05 \pm 0.45 \mathrm{kpc}$, $\Omega_{\odot}=31.09 \pm 0.78 \mathrm{~km} \mathrm{~s}^{-1} \mathrm{kpc}^{-1}, \alpha=0.022 \pm 0.029$, and $\left(U_{\mathrm{src}}, V_{\mathrm{src}}, W_{\mathrm{src}}\right)=(1.0 \pm 1.5,-14 \pm$ $2,-1.4 \pm 1.2)$. The Galaxy center distance $R_{0}$ is slightly smaller than the IAU standard of $8.5 \mathrm{kpc}$, but basically consistent within error bars. This value is also consistent with recent determinations of $R_{0}$ such as using stellar orbits around the $\operatorname{Sgr} \mathrm{A}^{*}[3,4] R_{0} \sim 8.3 \mathrm{kpc}$, and using Period-Luminosity relation for Mira [14], $R_{0} \sim 8.2 \mathrm{kpc}$. On the other hand, the angular velocity of the $\operatorname{Sun}\left(\Omega_{\odot} \equiv \Omega_{0}+\right.$ $\left.V_{\odot} / R_{0}\right)$ is somewhat larger than the IAU standard. For instance, if we adopt recent determination of $V_{\odot}=12 \mathrm{~km} \mathrm{~s}^{-1}$ [23], the Galactic rotation velocity at the Sun becomes $\Theta_{0}=238 \pm 14 \mathrm{~km}$ $\mathrm{s}^{-1}$. This indicates that the Galactic rotation velocity needs an upward modification from the IAU standard of $220 \mathrm{~km} \mathrm{~s}^{-1}$. We note that our determination of $\Omega_{\odot}$ is consistent with the proper motion measurement of Sgr A* [20], which also suggested an upward modification of $\Theta_{0}$. 


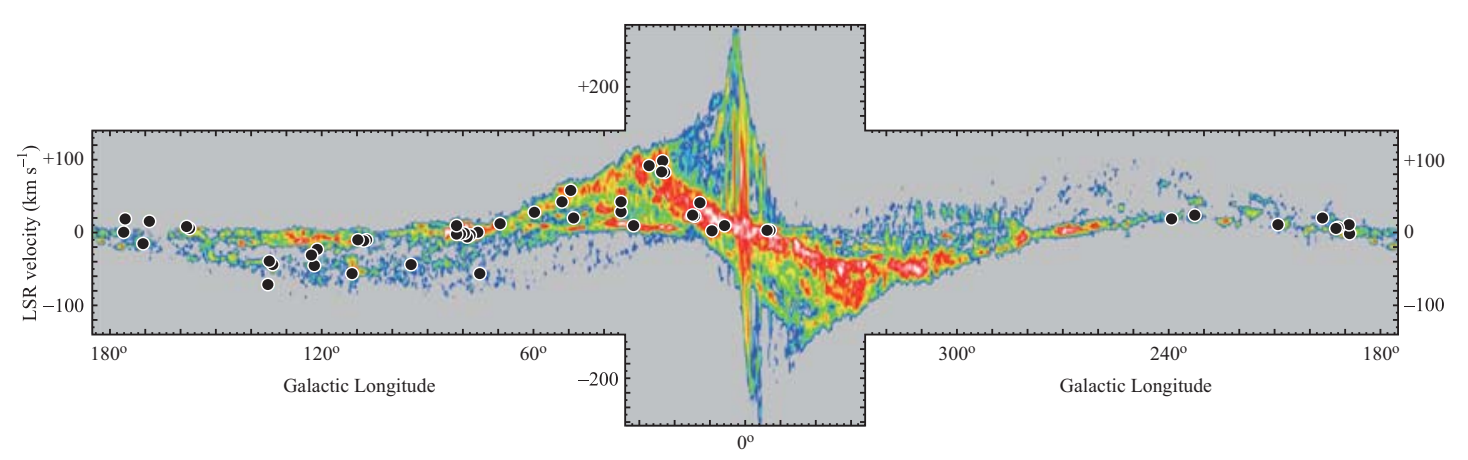

Figure 2: Distribution of 52 maser sources observed with VERA, VLBA and EVN on a CO longitudevelocity diagram. The CO map is from [2]
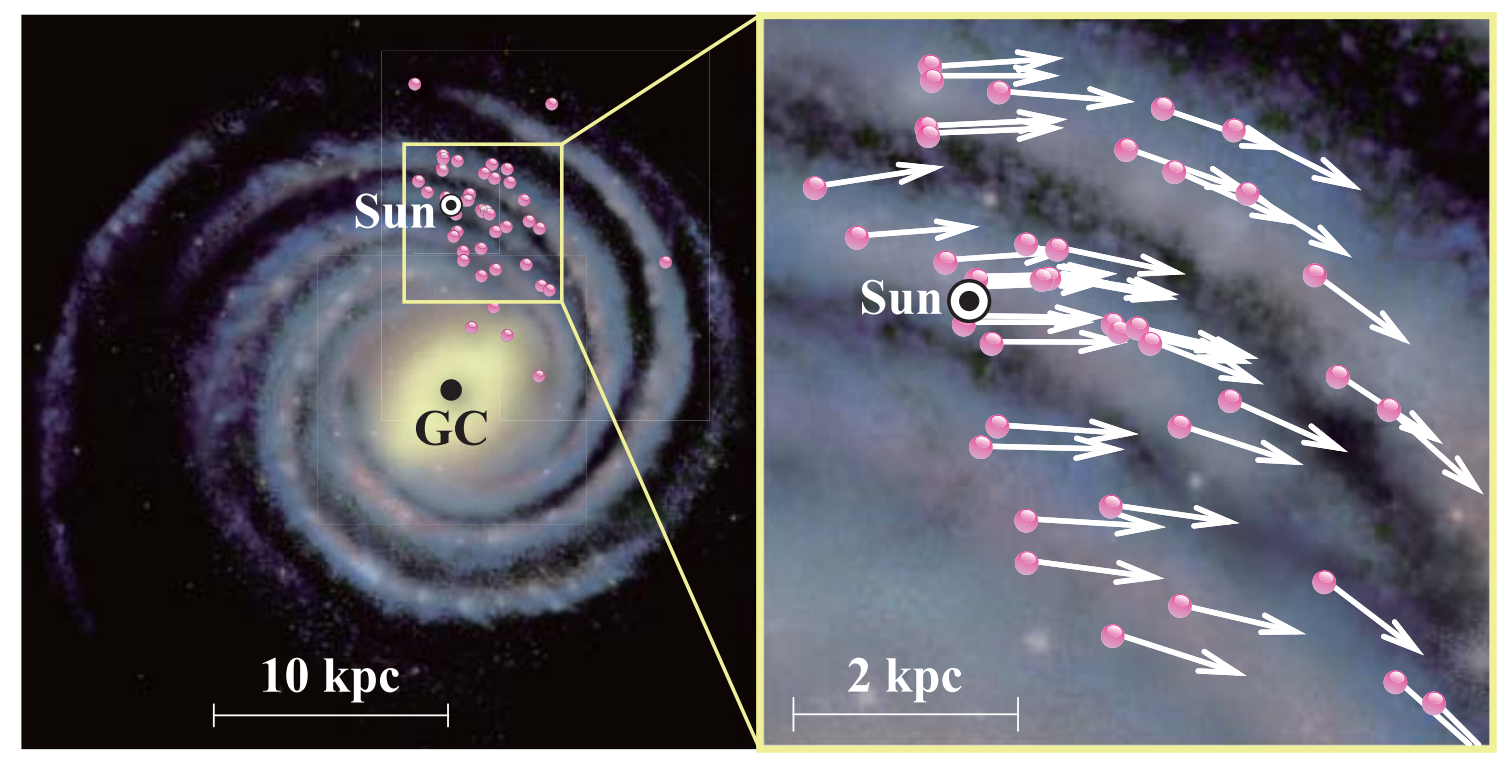

Figure 3: A schematic face-on view of the Milky Way Galaxy with locations of 52 star-forming regions for which astrometric results are obtained with VERA, VLBA or EVN. Right panel shows the proper motion vectors of these sources, which clearly show the Galactic rotation.

The increase of $\Theta_{0}$ also impacts on the mass distribution in the Galaxy and hence dark matter study. The Galactic mass is basically determined through the relation of $M=R \Theta^{2} / G$, where $R$ is the Galactic radius, $\Theta$ is the Galactic rotation velocity, and $G$ is the gravitational constant. Hence, $10 \%$ increases of $\Theta$ causes $\sim 20 \%$ increase of the mass of the Galaxy. Since the luminous mass (such as based on star counts) is not affected by the results in the present study, this indicates that within the Solar circle the Milky Way Galaxy contains more dark matter than previously expected. This result has a significant impact on direct dark matter search on the Earth: there are several ongoing experiments which attempt to directly detect possible dark matter particles falling onto the Earth, and the expected flux of dark matter particles is strongly dependent on the Galactic rotation velocity $\Theta_{0}$. 


\section{Future prospect}

VERA will continue astrometry of Galactic maser sources for next $\sim 10$ years. By completing astrometry of $\sim 30-40$ sources per year, it is expected that distances and proper motions will be available for $300-400$ sources by $\sim 2020$. In parallel with continuing regular astrometric observations, several possibilities of array extension are considered. First, in order to increase the sensitivity of VERA array, wideband recording system (up to $4 \mathrm{Gbps}$ ) is now under development. First fringe is already detected with the $4 \mathrm{Gbps}$ system, and it is anticipated that $4 \mathrm{Gbps}$ is in regular operation around 2014. Since the current data rate is $1 \mathrm{Gbps}, 4 \mathrm{Gbps}$ recording system will double the sensitivity of the VERA array. Moreover, addition of antennas will increase array sensitivity and also imaging capability. Japanese VLBI Network (JVN), which is a combined array of VERA and radio telescopes operated by universities (Yamaguchi 32m, Ibaraki 32m etc.), is an example of the array extension in Japan. Also, Korean VLBI Network (KVN) has recently been completed in South Korea, which has three of $21 \mathrm{~m}$ diameter telescopes operating at 22-129 GHz. KVN and VERA are now in collaboration to build a combined array which consist of seven of $20 \mathrm{~m}$-class antennas. In addition, there is a plan to establish EAVN (East Asian VLBI Network) by combining stations in Japan, Korea and China. For doing this, SHAO 25m and new 65m telescopes (and others as well) in Chinese VLBI Network are possible stations contributing to EAVN from China. With these possible extensions, we will continue operation for next 10 years, and hopefully combination of VERA and VLBA/BeSSeL data (and also Gaia) will be of great help for understanding the details of the Galactic structure such as fundamental parameters and also spiral structures.

Also important for future is to broaden the science cases of VERA. It is obvious that radio astronomy in next 10 years will be in "ALMA era", and hence it is important to consider the synergy between VLBI and ALMA. Clearly ALMA will provide angular resolution comparable with VLBI ( $\sim$ mas scale), and most importantly ALMA can obtain the thermal view of target sources which can never be obtained with VLBI. Therefore, combination of ALMA and VERA is expected to be a very powerful tool to study the details of maser emitting sources such as star-forming regions and AGB stars. A good demonstration of this has been recently conducted by [7], in which a new $\mathrm{H}_{2} \mathrm{O}$ maser at $232 \mathrm{GHz}$ was discovered in Orion source I based on combination of $22 \mathrm{GHz} \mathrm{H}_{2} \mathrm{O}$ and $43 \mathrm{GHz} \mathrm{SiO}$ maser observations with VERA and new ALMA observations at $232 \mathrm{GHz}$.

\section{References}

[1] Brunthaler A., et al., 2013, in this volume

[2] Dame T., et al., 2001, ApJ, 547, 792

[3] Ghez A., et al., 2008, ApJ, 689, 1044

[4] Gillessen S., et al., 2009, ApJ, 692, 1075

[5] Hirota T., et al., 2007, PASJ, 59, 897

[6] Hirota T., et al., 2011, PASJ, 63, 1

[7] Hirota T., et al., 2012, ApJ, 757, L1

[8] Hirota T., et al., 2013, in this volume 
[9] Honma M., et al., 2007, PASJ, 59, 889

[10] Honma M., et al., 2008, PASJ, 60, 935

[11] Honma M., et al., 2012, PASJ, 64, 136

[12] Imai H., et al., 2012, PASJ, 64, 142

[13] Kamezaki, et al., 2012, PASJ, 64, 7

[14] Matsunaga N., et al., 2009, MNRAS, 402, 934

[15] Motogi K., et al., 2013, in this volume

[16] Nagai H., et al., 2013, PASJ, 65, 24

[17] Niinuma K., et al., 2013, in this volume

[18] Orienti M., et al., 2013, MNRAS, 428, 2418

[19] Oyama T., et al., 2013, in this volume

[20] Reid M. J. et al., 2004, ApJ, 616, 872

[21] Sakai N., et al., 2012, PASJ, 64, 108

[22] Sawada-Satoh S., et al., 2013, in this volume

[23] Schönrich, et al., 2010, MNRAS, 403, 1829

[24] Sugiyama K., et al., 2013, in this volume 